\title{
ABSTRACTS
}

\section{MOTIVATIONAL INFLUENCES ON EPISTEMIC OBSERVATION}

\author{
by \\ Kayoko Inagaki \& Giyoo Hatano \\ Ochanomizu Women's University Dokkyo University
}

The present study aimed at investigating motivational influences on epistemic observation of a physical phenomenon. Two experiments, which followed Pre-test-Instruction-Post-test paradigm, were undertaken. Ss of 2 experimental groups were motivated for observation by receiving information discrepant with their prior beliefs.

One hundred and twenty-five 3rd-graders served as Ss of the 1st experiment. In the Instruction session, they observed and confirmed by a scale conservation of weight under deformations of a clay ball, and under changes of man's posture. Immediately before the observation, pupils of a group termed "Discrepant Information Group" (DI) were shown a table of response frequencies about conservation by an experimenter. Distribution of responses, which were pretended to be opinions of pupils of another, was markedly differnt from theirs, Ss of a "Discussion Group" (D) were required to anticipate conservation or non-conservation and to debate on the question. Control Group (C) Ss were given neither of these experimental manipulations.

The results were as follows:

1) Ss who observed the event after incongruity was aroused (as in DI) could recognize the event more accurately and could more readily generalize the principles of conservation.
2) If Ss were strongly committed to a certain belief (as in D), they often conceptualized ambiguous results in a biased manner, making them consistent with the belief.

As to generality of learning, however, performance of D Ss did not differ significantly from that of C Ss. This result was an unexpected one. Its interpretation was that there was low incongruity in $\mathrm{D}$, because proponents of conservation were highly predominant at the discussion.

In order to verify the interpretation mentioned above, the 2nd experiment was undertaken. Eightyseven 4 th-graders observed and confirmed by a scale conservation of weight under dissolution of sugar into water. One school class was assigned to $\mathrm{C}$. Two groups of 22 pupils, of whom $2 / 3$ were nonconservers, were selected from 2 other classes and served as D. They were expected to experience high incongruity during the discussion.

The results showed that, compared with $\mathrm{C}$, improvement of performance was greater among D. They could state adequate explanations of conservation of weight, generalize more readily the principles of conservation, and resist extinction (the observation of an apparently non-conserving event). Furthermore, Ss of D reported high epistemic curiosity. 